\title{
1 Exploring the current role and future role of the pharmacists in osteoporosis screening
}

2

3

4

5

6

7

8

9

and management in Malaysia.

\section{Introduction}

Osteoporosis inflicts a substantial burden on public health and national economies on the account of fragility fractures that results from the condition [1]. By year 2050, 50\% of hip fractures worldwide will occur in Asia [2]. As such, it is imperative to conduct an intervention for the early detection and treatment of patients at risk $[1]$.

The pharmacy profession has shifted from a product-centred approach to a more patient-oriented approach, which requires more direct interventions and involvement by the pharmacist going beyond medication dispensing [3]. This practice change has led to more intervention by pharmacists [4-7]. There is a growing body of literature supporting the roles of pharmacists in osteoporosis [4-8]. Studies conducted in various settings around the globe have shown that these pharmacists' interventions improved adherence to osteoporosis medication. Some studies have also reported improvements in both clinical and economic outcome [4-7].

Most pharmaceutical care services are mainly targeted at the treatment of osteoporosis. A further literature search revealed that there are three randomized control trials (RCTs) conducted overseas by community pharmacies to evaluate the impact of pharmacist's interventions on osteoporosis management [9-11]. However, two of these studies were considered biased [12]. The study by Crockett et al had a high risk of both selection and information bias as self-reported assessment was used [10, 12]. As for the study by McDonough et al the study suffered from a high risk of selection bias as the recruitment size and followed up differed between groups $[11,12]$. The third study by Yuksel et al demonstrated low bias in both aspects $[9,12]$. Nonetheless, all three studies provided attestation that the intervention of pharmacists increased the number of patients that had their BMD tested and calcium intake initiated, indicating that pharmacists may have a role to play in reducing the gap in osteoporosis management [9-11].

Foundational research on the pharmacist role in osteoporosis screening and management in the literature is sparse. Integrating a pharmacist into osteoporosis screening and management is a novel concept and requires a new model of practice to be explored. As such, investigating the perspective of key stakeholders on the 
pharmacists' role in osteoporosis screening and management is potentially crucial to the successful implementation of this concept. To date, there have been no studies on a pharmacist-led osteoporosis screening programme in Malaysia. This study explores the perspective of stakeholders such as policy makers, doctors, pharmacists, nurses and patients towards the role of pharmacists in osteoporosis screening and management.

\section{Aim of the study}

The aim of this study was to explore the perspective of stakeholders such as policy makers, doctors, pharmacists, nurses and patients towards the role of pharmacists in osteoporosis screening and management.

\section{Ethics approval}

Ethical approval was obtained from the University Malaya Medical Centre Ethics Committee (approval number: 914.14).

\section{Method}

Qualitative, semi-structured in-depth interviews were used to allow for a detailed exploration of the perspective of stakeholders such as patients, nurses, doctors, pharmacists and policy makers on the role of the pharmacists in osteoporosis screening and management.

This study was conducted at the primary care clinics located within a teaching hospital in Malaysia. The data was collected from October 2012 to January 2013. Participants were selected via purposive sampling. Nonosteoporotic postmenopausal women $\geq 50$ years of age from three main ethnics groups (Malays, Chinese, and Indians) in Malaysia were selected to account for any cultural differences. Healthcare professionals (pharmacist, primary care doctors, and nurses) with more than one year of working experience at the primary care clinic were selected as they would have sufficient working experience with the primary care clinic system. Policy makers were defined as individuals working in the upper management of the tertiary hospital. This includes head of departments who have an authority to influence and decide to change the practice

Patients were recruited by approaching them in the primary care clinic while they were waiting for their doctors' appointment. Patients' medical records were screened to confirm that they did not have osteoporosis/osteopenia. Healthcare professionals and policy makers were recruited by approaching them personally and setting up 
appointments for the interviews. The purpose of the study was explained to the participants using written information and consent was obtained.

A topic guide was used to guide the interviews. Three topic guides were developed in English: one for patients, one for healthcare professionals and one for policy makers. The development of the topic guides was based on literature and an expert panel consisting of a consultant endocrinologist and four pharmacists. A translated topic guide was needed as some interviews were conducted in Malay. Forward and backward translation were conducted by two bilingual pharmacists who were not participants of this study. Differences were discussed using the expert panel. Each topic guide was piloted for finalization.

All patients, healthcare professional and policy maker interviews were conducted by the researcher (LST), who was a pharmacist. However, this was not revealed to the participants until the interview had ended. Interviews lasted an average of 60 minutes and were conducted by a trained researcher in either English or Malay. All interviews were transcribed verbatim and checked by a second researcher/pharmacist for accuracy. All transcripts were offered to each interviewee to check for accuracy but all declined.

\section{Data Analysis}

QSR International Pty Ltd. NVivo version 10 for Windows, 2012 was used to aid in the analysis of the data. Analysis began during data collection. At the end of each interview, the researcher wrote memos of interesting topics that were raised in the interview. The data analysis was data-driven.

Thematic analysis was used to analyze the interview data [13, 14]. It involves analyzing the data as a whole to find repeated patterns of meaning $[13,14]$. The analysis of the data involved repeatedly reading the transcripts while listening to the audio recording, and emerging topics were coded and constantly compared and contrasted with other transcripts by one researcher developing a coding framework [13].

The 'one sheet of paper' (OSOP) analysis as described by Ziebland and McPherson (2006) was then used to progress the analysis of the data. Emerging themes were then refocused at a broader level of themes [13]. This involved sorting the themes into broader themes and collating all the relevant coded data extracts within the identified theme. OSOP involved reading through each code and then noting on a piece of paper all issues that 
were raised and making connections between them [15]. This process allowed identification of deviant cases that did not fit into the emerging story. These deviant cases were then reanalyzed and accounted for in the analysis [16]. Data saturation was reached when no new codes emerged when new participants were recruited into the study [17].

The analysis of each stakeholder: patients, pharmacists, nurses, doctors and policy makers were conducted separately. However, the themes which emerged within each group of stakeholder were similar and the analysis were combined. As a form of validation, a subset of transcripts was reviewed by two other members of the research team for agreement of the codes and themes.

For verification purposes, all transcripts were translated to English. However, the original language was used during data analysis. Analyzing in the original language facilitated cross checking the data with the audio recordings. During data analysis, selected themes and sub-themes from the Malay language were translated to English. The study by Chen \& Boore supports that verbatim transcripts and data analysis to be conducted in the original language and only emergent concepts, themes and sub-themes needed translation to English [18].

\section{Results}

Fifty-six participants were interviewed (patients $=20$, nurses $=10$, pharmacists $=11$, doctors $=10$ and policymakers $=5$ ). Each interview lasted an average of $60 \mathrm{~min}$. Patients interviewed were 52-72 years of age. Six patients were Malay, six were Indians, and eight were Chinese. All nurses had four years or more of working experience. Pharmacists' work experience ranged from 2 to 4 years, whilst doctors' work experience ranged from 3 to 4 years.

Most of the interviews were conducted in English, sixteen patients' interviews, two nurses' interviews, nine pharmacists' interviews, and four doctors. Two patient interviews, eight nurses' interviews, two pharmacist interviews and five interviews with policy makers were conducted in both English and Malay. Two patient interviews were conducted in Malay.

The results were divided to two sections: the current role of the pharmacists and the future role of the pharmacists in osteoporosis screening and management 
The current perceived role of the pharmacists in practice

123 Pharmacists were principally perceived by participants to be suppliers of medication, although there was some recognition of roles in providing medication safety, medication costing and medication advice [Table 1].

Table 1: Summary of current perceived pharmacists' role by patients, nurses, doctors, pharmacists and policy makers

\begin{tabular}{ll}
\hline Current perceived pharmacists role & Sub themes \\
\hline Suppliers of medication & Dispensing of medications \\
& Approval of medication supply \\
Medication safety & Ensure patients receives the appropriate medications \\
Medication advice & Medication advice to patients \\
& Medication advice to other healthcare professionals \\
Medication costing & Budgeting of medication fund
\end{tabular}

128

Suppliers of medication

Dispensing medications

The supplying of medications by dispensing was seen to be the core duty of the pharmacists. Dispensing was perceived to be an activity where the pharmacists receives a prescription from the patients at the counter and supplies the appropriate medication. If it is a repeated prescription, the pharmacists would set another appointment date for the patients to collect the medication.

"So I take the (medication from the pharmacist for the) first time... (then) they give me another date to, replenish... (my medication in about) six months or one year... appointment. That's all..."

Approval of medication supply

141 Additionally, the supplying of medications also refers to the pharmacists' role in the hospital's Drugs and

142 Therapeutics sub-committee whereby policies about medication usage are determined. Various policies are approved to only allow certain group of specialist to prescribe certain medications. For example, osteoporosis 
medications can only be dispensed if a bone mineral densitometry (BMD) scan indicates osteoporosis and if it is

145 prescribed by endocrinologists, orthopaedics and gynaecologists. Therefore, the pharmacists would need to

146 ensure the appropriate forms and procedures are conducted before the medication can be dispensed to the

147 patients.

148

149 "So the pharmacists will assess if the patients can get the medication for free, whether the doctor can prescribe 150 the medication or not. Because previously, Fosamax we had to attach the BMD report."

(Nurse-9/Female/42 years)

Medication safety

Ensure patients receives the appropriate medication

The pharmacists were seen to be the final safety net before the patients take home their medications. As the primary care clinic has a lot of trainee doctors, the pharmacists' role to ensure that the patients receive the appropriate medication is crucial. To elaborate on this, the pharmacists play an important role to check the appropriateness of the medication in terms of the indication, dose and interaction.

"... At the moment the role... (is) making sure that... the med(ication), the patient is receiving is safe... The main focus is safety... whatever prescription that come in... (we ensure the) dose, the combination of products... is safe for the patient(s)..."

(Pharmacist-8/Female/29 years)

163

Medication advice

Medication advice to patients

166 The pharmacists were also recognised for their role in giving medication advice. The pharmacists would

167 dispense the medications and provide information to the patients regarding the medications. The information

168 provided includes the indication, mode of action, side effects and method of taking the medications. The monitoring of the patients adherence and compliance is also part of this process. There were some patients who recognized the pharmacists' role in advice for minor ailments and supplements. 
"Pharmacist, I think it's very important... the role would be to explain to the patients regarding the indication of the medication, the mode of action and the proper way of taking the medications."

(Doctor-3/Female/36 years)

\section{Medication advice to other healthcare professionals}

Apart from that, pharmacists were seen by the other healthcare professionals such as doctors and nurses to be medication experts. They would seek advice from the pharmacists regarding: side effects, interactions, dosage, approval to prescribe and availability. However; this is not a common occurrence.

"But for me... the pharmacist relationship is... just to ask about the drugs side effects, the drugs whether (it) can be prescribe, about the dosage, everything..."

(Doctor-8/Female/29 years)

\section{Medication costing}

Budgeting of medication fund

Pharmacists were seen to be involved in medication costing via the hospital's Drugs and Therapeutics subcommittee. The policy to only allow certain groups of specialist to prescribe certain medications is part of fund management. Due to the shortage of funds there was a shortage of medications. Hence, pharmacists at the upper management level would need to develop policies to ensure sufficient medication is available where else pharmacists at the frontline would need to ensure these policies are adhered too.

"(This policy is to) save cost because we have to ensure that the usage of the medication is not too high. Hence, we limited it to a certain amount of patients (whom are under the specialists' care). Therefore, we are forced to do screening of prescribed medication this way."

(Policy maker-5/Male/44 years)

\section{The perceived future role of the pharmacists in osteoporosis screening and management}

Doctors, nurses, patients, policy makers and pharmacists themselves were eager for pharmacists to expand their role beyond medication: supply, advice, costing and safety. The stakeholders perceived that the pharmacists should expand their role in terms of counselling, creating awareness and screening of osteoporosis [Table 2]. 
"But I think we are actually well position to actually do... this screening and in fact to do the counsel(ing) (and)

Table 2: Summary of perceived future pharmacists' role by patients, nurses, doctors, pharmacists and policy makers

\begin{tabular}{l}
\hline Perceived future pharmacists role \\
\hline Counselling \\
Creating osteoporosis awareness \\
Screening of osteoporosis \\
\hline
\end{tabular}

Counselling

Counselling was seen to be conducting activities such as the current medication therapy adherence and compliance (MTAC) clinic conducted for diabetic patients. This was an individualized service provided by the pharmacists. Pharmacists assist the patients in adjusting their insulin dose and give lifestyle advice. Recommendations to doctor regarding therapy were also given if necessary. However, this service is only conducted for diabetics and patients on warfarin. Therefore, stakeholders noted the possibility of this kind of services to be extended to osteoporosis and other diseases. Additionally, group counselling by the pharmacists was also suggested.

"I want the MTAC [clinic for] osteoporosis to be implemented again in our hospital after proper planning... because... from here we can... reach out to the public because... my daily job. I think (it) is very... difficult for me to actually talk to them (patients)."

(Pharmacist-10/Female/28 years)

Creating awareness of osteoporosis

The second area suggested was creating awareness on osteoporosis and public health in general. Stakeholders suggest various ways such as creating posters, campaigns or giving health talks during clinic session. However, this could also be done opportunistically. For example, pharmacists could casually mention to a postmenopausal women if she has undergone a BMD scan. Pharmacists were seen to be most accessible to patients at the community level. Therefore, pharmacists are in an ideal position to create awareness on osteoporosis and various diseases. 
228 "I think they (primary care pharmacists) have (a) big role because... they are more... involved with community...

229 they have a major role in screening, not only osteoporosis, other diseases as well. And then to educate patients

(Doctor-5/Male/30 years)

Screening of osteoporosis

Lastly, the pharmacists were seen to be in an ideal position to screen for osteoporosis. This is because

pharmacists were seen to be more accessible. Patients would visit the pharmacists several times before their next doctor's appointment for their repeat prescriptions. This gives the pharmacists the opportunity to tap into screening and prevention of osteoporosis. Patients also perceived pharmacists to be knowledgeable and trust pharmacist for advice.

"Pharmacist can explain to us... rheumatism (referring to osteoporosis)... what you (kind of supplements to)

(Patient-6/Female/72 years)

242 Currently, both the doctors and nurses are unable to screen for osteoporosis systematically due to the time constraint. If pharmacists were involved in osteoporosis screening it was seen as an improvement to the healthcare system. This facilitates the healthcare professionals to understand each others' scope of practice better leading to a more effective healthcare system. Additionally, the involvement of pharmacists in osteoporosis screening would lighten the workload of doctors and nurses. This in turn saves both the patients and healthcare professionals' time. The pharmacist would screen for osteoporosis and the doctors would focus on diagnosis and treatment. Hence, the pharmacists could play a part in osteoporosis screening alerting the doctors when a BMD scan may be needed. This will assists in detecting untreated osteoporosis.

“...Pharmacist can help to save (the) doctor's time... because some patient(s) (do not) need to (be) referred (to the) doctor. Waste both... (the) doctors' and patients' time... So if (the) pharmacists can do that (osteoporosis expansion of the pharmacist role in osteoporosis screening. They felt that they weren't contributing enough to 

pharmacist non-dispensing role in osteoporosis screening.

"(We) dispense like (a) robot... you just push, push, push the thing (medication) out."

Lastly, all the participants concurred that the expansion of the non-dispensing role of the pharmacists to osteoporosis screening was seen as progression for the profession. The pharmacists' skills were considered underutilized and shifting from a more medication-centred approach to a more patient-orientated approach was suggested. This emphasizes the need to expand the pharmacists' role to osteoporosis screening. Additionally, pharmacists were well equipped with the knowledge on the disease, treatment and prevention. Stakeholders noted the success of pharmacists' independent prescribing role overseas such as in the UK. They unanimously agreed that the pharmacists' role should be expanded to osteoporosis management.

“(The pharmacists') job scope is expanding all this time... I wouldn’t be surprised if pharmacist (start) screening (for osteoporosis) since (there are) all ready... pharmacist prescribers (overseas)...”

\section{Discussion}

The semi-structured interviews outlined above gives us a unique insight into the opinions and views of policy makers, doctors, pharmacist, nurses and patients around the current role of pharmacist in Malaysia and perspective of their future role in osteoporosis management. These stakeholders perceived the current pharmacist role to be medication centred: medication supplier, medication advice, medication safety and medication costing. However, the doctors, nurses, patients, policy makers and pharmacists themselves were eager for pharmacists to expand their role beyond medication to more patient centred such as counselling, creating awareness and screening of osteoporosis. such as dispensing and compounding of medication shifts to a strengthening role of providing medication information as well as improving the quality use of medication activities such as medication management 
reviews and chronic disease management programs [19]. In Australia, pharmacists are viewed as highly trained

288

289

290

291

292

293

294

295

296

297

298

299

300

301

302

303

304

305

306

307

308

309

310

311

312

313

314

315 yet underutilised and there is growing support to extend the role of pharmacist within the primary health care sector [20]. Similarly, the stake holders cited that pharmacists were seen to be more accessible to screen for osteoporosis and that they were underutilized.

Recent research on the expansion of the pharmacist role has shown positive response from general practitioners recognising the potential benefit of integrating a pharmacist into the medical team with the recognised benefits perceived by the general practitioners increasing over time [21-23]. A systematic review in 2014, revealed that pharmacist co-located in general practice clinics could deliver favourable results when conducting chronic disease clinics and quality use of medicines reviews, resulting in improved clinical outcomes such as blood pressure, blood sugar and cholesterol reduction [24]. Research also found that patients are generally supportive of a pharmacist involvement in non-dispensing roles [25].

Our study noted that one of the pharmacist future role could be to create awareness of osteoporosis._This was a similar finding from another Malaysian study exploring community pharmacist role in type two diabetes management. However, creating awareness of diabetes was a role that the pharmacist was currently conducting [26]. As such, there is a potential for pharmacists to create awareness in osteoporosis as well as other chronic diseases such as diabetes.

Additionally, our study also found that Malaysian pharmacists were minimally involved in osteoporosis management. These findings were similar to another Malaysian study in community pharmacists where the pharmacists appeared to be rooted in the traditional role of medication dispensing but recognizing the potential to expend their roles. In that study, only ad-hoc counselling, such as advice on osteoporosis prevention, risk factors and lifestyle medication was practiced [27]. Our interviewed pharmacists referred to their current role as 'robotic dispensers' and unanimously agreed for an expansion of the pharmacist role in osteoporosis screening. The themes from this study resonates with the themes that emerged from other studies. The Canadian survey reported that although pharmacists spend most of their time on dispensing duties but over $60 \%$ believed that the time had come to expand their role in areas such as disease prevention and health promotion [28]. 
316 The interpretation of the results may be limited to the confined area where the participants were recruited. As

317 such, this qualitative research was then able to reflect on the local setting and are not generalizable to other

318 setting. Nonetheless, the themes generated resonated with themes that occurred in other studies. Another

319 limitation of the study is that the interviews were only conducted in Malay or English, which are the two main

320 languages, spoken in Malaysia. Interviews were not conducted in Mandarin or Tamil, as those proficient in

321 Mandarin or Tamil would also be able to speak in English or Malays. Although, the data was not analysed

322 independently by two researchers, sections of the coded transcripts were presented to two researchers on

323 separate occasions to establish if other members of the research team agreed with the themes assigned.

324 Discussions were conducted until a consensus was reached. Additionally, the healthcare professionals had varying years of working experiences ranging from two to four years for doctors and pharmacists and more than four years for nurses. However, their views towards the pharmacists role were similar.

327

The strength of this study is that the sample comprised of a purposive sample of healthcare professionals and consumers involved in the primary care setting. Hence, we were able to gain an in depth understanding of the perceived current and future role of the pharmacist in osteoporosis screening and management. Another strength of this study is that it used qualitative methods to contribute to the currently sparse literature on stakeholders' perception towards the pharmacists' role in osteoporosis screening and management. The findings from this study has the potential to highlight the successful integration of the non-dispensing role of the pharmacist in osteoporosis screening and management through an understanding of the current position of the pharmacist and the expected direction of the pharmacy profession. Future studies should focus on developing and integrating the non-dispensing pharmacist role into daily clinical practice and then assessing for effectiveness.

\section{Conclusion}

Although the current role of pharmacists in Malaysia is medication-centred, healthcare professionals and consumers are willing to expand it to non-dispensing roles, in particular to osteoporosis screening and management. This encourages further efforts to examine the potentially missed opportunities for Malaysian pharmacist to assume an expanded role in health care.

Conflicts of interest: All authors declare no conflict of interest. 
Funding: Ministry of Science, Technology and Innovation (MOSTI) fund (06-02-12-SF0183).

1. Kanis JA. WHO Scientific Group Technical Report: Assessment of osteoporosis at the primary health care level. UK. WHO. 2007:1-339. https://www.sheffield.ac.uk/FRAX/pdfs/WHO_Technical_Report.pdf. Accessed 08 Aug 2017

2. Cooper C, Campion G, J ML. Hip fracture in the elderly: A world-wide projection. Osteoporos Int. 1992;2:285-9.

3. Schneider JK, Nickman NA. Assessment of pharmaceutical care needs in an ambulatory setting. Hosp Pharm. 1994;29:238-42.

4. Van Boven JFM, Stuurman Bieze AGG, Hiddink EG, Postma MJ, Vegter S. Medication monitoring and optimization: A targeted pharmacist program for effective and cost-effective improvement of chronic therapy adherence. J Manag Care Spec Pharm. 2014;20(8):786-92.

5. Stuurman-Bieze AGG, Hiddink EG, van Boven JFM, Vegter S. Proactive pharmaceutical care interventions decrease patients' nonadherence to osteoporosis medication. Osteoporos Int. 2014;25:1807-12. 6. George PP, Molina JAD, Cheah J, Chan SC, Lim BP. The evolving role of the community pharmacist in chronic disease management- A literature review. Ann Acad Med Singapore. 2010;39:861-7.

7. Lai PSM, Chua SS, Chan SP. Impact of pharmaceutical care on knowledge, quality of life and satisfaction of postmenopausal women with osteoporosis. Int J Clin Pharm. 2013;35(4):629-37.

8. Toh LS, Lai PS, Othman S, Wong KT, Low BY, Anderson C. An analysis of inter-professional collaboration in osteoporosis screening at a primary care level using the D'Amour model. Res Social Adm Pharm. 2016; doi: 10.1016/j.sapharm.2016.10.004.

9. Yuksel N, Majumdar SR, Biggs C, Tsuyuki RT. Community pharmacist-initiated screening program for osteoporosis: randomized controlled trial. Osteoporos Int. 2010;21(3):391-8.

10. Crockett JA, Taylor SJ, McLeod LJ. Patient responses to an integrated service, initiated by community pharmacists, for the prevention of osteoporosis. Int J Pharm Pract. 2008;16(2):65-72.

11. McDonough RP, Doucette WR, Kumbera P, Klepser DG. An evaluation of managing and educating patients on the risk of glucocorticoid-induced osteoporosis. Value Health. 2005;8(1):24-31.

12. Elias MN, Burden AM, Cadarette SM. The impact of pharmacist interventions on osteoporosis management: a systematic review. Osteoporos Int. 2011;22(10):2587-96.

13. Braun V, Clarke V. Using thematic analysis in psychology. Qual Res Psychol. 2008;3(2):77-101.

14. Boyatzis RE. Transforming qualitative information: Thematic analysis and code development.

Thousand Oaks, California: Sage publications; 1998.

15. Ziebland S, McPherson A. Making sense of qualitatative data analysis: An introduction with illustrations from DIPEx (personal experiences of health and illness). Med Educ. 2006;40:405-14.

16. Bazeley P. Analysing qualitative data: More than 'identifying themes'. Malaysian Journal of Qualitative Research. 2009;2:6-22.

17. Bowen GA. Naturalistic inquiry and the saturation concept: a research note. Qual Res. 2008;8(1):137-

52.

18. Chen HY, Boore JRP. Translation and back-translation in qualitative nursing research: Methodological review. J Clin Nurs. 2009;19:234-9.

19. Pharmacy Guild Australia. Medication Management Review Program. 2010.

http://www.guild.org.au/mmr/content.asp?id=406. Accessed 08 Aug 2017

20. Australia Department of Health and Ageing WA. Primary healthcare reform in Australia: report to support Australia's first national primary healthcare strategy. Western Australia. Dept of Health and Ageing; 2009.

21. Farrell B, Pottie K, Woodend K, Yao V, Dolovich L, Kennie N, et al. Shifts in expectations: evaluating physicians' perceptions as pharmacists become integrated into family practice. J Interprof Care. 2010;24(1):809 .

22. Blondal AB, Jonsson JS, Sporrong SK, Almarsdottir AB. General practitioners' perceptions of the current status and pharmacists' contribution to primary care in Iceland. Int J Clin Pharm. 2017; 39 (4): 945-952. 23. Tan EC, George J, Stewart K, Elliott RA. Improving osteoporosis management in general practice: a pharmacist-led drug use evaluation program. Drugs Aging. 2014;31(9):703-9.

24. Tan EC, Stewart K, Elliott RA, George J. Pharmacist services provided in general practice clinics: a systematic review and meta-analysis. Res Social Adm Pharm. 2014;10(4):608-22.

25. Iversen L, Mollison J, MacLeod TN. Attitudes of the general public to the expanding role of community pharmacists: a pilot study. Fam Pract. 2001;18(5):534-6. 
26. Lee EL, Wong PS, Tan MY, Sheridan J. What role could community pharmacists in Malaysia play in diabetes self-management education and support? The views of individuals with type 2 diabetes. Int J Pharm Pract. 2017; doi: 10.1111/ijpp.12374.

406 27. Nik J, Lai PS, Ng CJ, Emmerton L. A qualitative study of community pharmacists' opinions on the provision of osteoporosis disease state management services in Malaysia. BMC Health Serv Res. 2016;16:448. 28. Jorgenson D, Lamb D, MacKinnon NJ. Practice change challenges and priorities: a national survey of practising pharmacists. Can Pharm J. 2011;144:125-31. 